\title{
Treatment of cardiomyopathy and rhabdomyolysis in long-chain fat oxidation disorders using an anaplerotic odd-chain triglyceride
}

\author{
Charles R. Roe, ${ }^{1}$ Lawrence Sweetman, ${ }^{1}$ Diane S. Roe, ${ }^{1}$ France David, ${ }^{2}$ \\ and Henri Brunengraber ${ }^{2}$
}

${ }^{1}$ Institute of Metabolic Disease, Baylor University Medical Center, Dallas, Texas, USA

${ }^{2}$ Department of Nutrition, Case Western Reserve University, Cleveland, Ohio, USA

\begin{abstract}
The current dietary treatment of long-chain fatty acid oxidation defects (high carbohydrate with medium-even-chain triglycerides and reduced amounts of long-chain fats) fails, in many cases, to prevent cardiomyopathy, rhabdomyolysis, and muscle weakness. We hypothesized that the apparent defect in energy production results from a depletion of the catalytic intermediates of the citric acid cycle via leakage through cell membranes (cataplerosis). We further hypothesized that replacing dietary medium-even-chain fatty acids (precursors of acetyl-CoA) by medium-odd-chain fatty acids (precursors of acetyl-CoA and anaplerotic propionyl-CoA) would restore energy production and improve cardiac and skeletal muscle function. We fed subjects with long-chain defects a controlled diet in which the fat component was switched from medium-even-chain triglycerides to triheptanoin. In three patients with very-long-chain acyl-CoA dehydrogenase deficiency, this treatment led rapidly to clinical improvement that included the permanent disappearance of chronic cardiomyopathy, rhabdomyolysis, and muscle weakness (for more than 2 years in one child), and of rhabdomyolysis and weakness in the others. There was no evidence of propionyl overload in these patients. The treatment has been well tolerated for up to 26 months and opens new avenues for the management of patients with mitochondrial fat oxidation disorders.
\end{abstract}

J. Clin. Invest. 110:259-269 (2002). doi:10.1172/JCI200215311.

\section{Introduction}

Since the identification of carnitine palmitoyltransferase (CPT) deficiency more than 25 years ago $(1,2)$, nearly every enzymatic step required for mitochondrial $\beta$-oxidation of saturated and unsaturated fatty acids has been associated with an inherited metabolic disorder (3). These disorders effectively pre-empt the use of dietary or body fat as an energy source. Clinical consequences of these diseases can range from no symptoms (rarely) to increasingly severe manifestations, including acute and chronic cardiomyopathy, intermittent rhabdomyolysis, hypoglycemia, peripheral neuropathy,

Received for publication February 20, 2002, and accepted in revised form June 1, 2002.

Address correspondence to: Charles R. Roe, Institute of Metabolic Disease, Baylor University Medical Center, 3812 Elm Street, Dallas, Texas 75226, USA. Phone: (214) 820-4533; Fax: (214) 820-4853; E-mail: charlesr@BaylorHealth.edu. Conflict of interest: No conflict of interest has been declared. Nonstandard abbreviations used: carnitine palmitoyltransferase (CPT); medium-chain triglyceride (MCT); $\beta$-hydroxybutyrate (BHB); $\beta$-hydroxypentanoate (BHP); $\beta$-ketopentanoate (BKP); acetoacetate (AcAc); citric acid cycle (CAC); very-long-chain acyl-CoA dehydrogenase (VLCAD); $\left[16-{ }^{2} \mathrm{H}_{3}\right]$ palmitate (C16); $\left[15-{ }^{2} \mathrm{H}_{3}\right]$ pentadecanoate $(\mathrm{C} 15) ;\left[9-{ }^{2} \mathrm{H}_{3}\right]$ nonanoate (C9); $\left[7-{ }^{2} \mathrm{H}_{3}\right]$ heptanoate (C7); L-3-hydroxy acyl-CoA dehydrogenase (LCHAD); mitochondrial trifunctional protein (MTP); cis-5-C14:1 (C14:1); left ventricular end-diastolic diameter (LVEDD); tetradecanoylcarnitine (C14); creatine phosphokinase (CPK); electron-transfer flavoprotein (ETF); octanoate (C8); propionylcarnitine (C3); acylcarnitine (AC). hypotonia, and sudden death. More confusing is the fact that the onset of symptoms in a given defect can be either neonatal and devastating, infantile with chronic disease, or delayed until adulthood. The extraordinary phenotypic variation within this group of disorders has made it difficult to diagnose them or to assess the efficacy of current therapy. The ongoing introduction of newborn screening by tandem mass spectrometry (4) should result in opportunities to evaluate benefits from existing treatment strategies as well as stimulate the development of new and more effective therapies. At this time, treatment includes restriction of dietary fat, avoiding fasting, suppressing lipolysis, use of dietary medium-chain triglycerides (MCTs), and carnitine supplementation.

Experience with patients with these disorders strongly suggests that current treatment strategies are limited in their ability to control or prevent serious symptoms (cardiomyopathy with associated arrhythmias and conduction defects, progressive peripheral neuropathy, retinopathy, rhabdomyolysis, or sudden death). The conclusion of a recent review of 107 patients representing nearly all of the fat oxidation defects was a strong warning that existing therapy is, for most of the defects, palliative or even ineffective $(5,6)$.

One common denominator to the pathophysiology of these disorders is the impairment of hepatic production of the ketone bodies $\beta$-hydroxybutyrate (BHB) 
and acetoacetate (AcAc) for export as a source of energy to other organs. Also, many of the symptoms and complications experienced by these patients can be viewed as the result of energy deprivation. This occurs despite ample supply of acetyl groups to the citric acid cycle (CAC) from dietary carbohydrates, and a normal respiratory chain. This led us to hypothesize that, at least in some cases, the CAC might be depleted of the catalytic units, which carry the acetyl groups as they are oxidized. This depletion would result from excessive leakage from the cells of intermediates of the CAC (cataplerosis). The normal mechanisms that refill the pools of catalytic intermediates of the CAC (anaplerosis) could not balance the excessive leakage. Leakiness of cell membranes is strongly suggested by the release of intracellular enzymes such as creatine kinase. To test this hypothesis, we examined the potential therapeutic benefit of the odd-carbon triglyceride triheptanoin. After hydrolysis of the latter, heptanoate is $\beta$-oxidized in the mitochondria of most tissues, bypassing the CPT system. Oxidation of heptanoate forms both acetyl-CoA and propionyl-CoA. PropionylCoA is an efficient anaplerotic substrate for the CAC. In the liver, heptanoate carbon is converted to the fivecarbon ketone bodies $\beta$-hydroxypentanoate (BHP; $\beta$-hydroxyvalerate) and $\beta$-ketopentanoate (BKP; $\beta$-ketovalerate), which are exported to peripheral tissues (7), including the brain, where they also generate acetyl-CoA and propionyl-CoA.

The remarkable outcome of this treatment strategy in three patients with the cardiomyopathic form of the verylong-chain acyl-CoA dehydrogenase (VLCAD) deficiency is presented as an example of the potential benefits of this treatment for a number of disorders of fat oxidation.

\section{Methods}

In vitro analysis of mitochondrial $\beta$-oxidation by tandem mass spectrometry

Fibroblast cell lines from three patients with VLCAD deficiency (proven by enzyme assay and/or blood acylcarnitine analysis) (8) and normal cell lines were incubated in triplicate in T-25 flasks with $3.5 \mathrm{ml}$ freshly prepared DMEM (GIBCO BRL; Invitrogen Corp., Carlsbad, California, USA) containing 10\% FCS, $0.4 \mathrm{mM}$ L-carnitine, and antibiotic/antimycotic. Each cell line was incubated separately with the following labeled fatty acids $(0.2 \mathrm{mM})$ bound to fatty acid-free BSA: $\left[16-{ }^{2} \mathrm{H}_{3}\right]$ palmitate (C16), $\left[15^{2} \mathrm{H}_{3}\right]$ pentadecanoate (C15), $\left[9-{ }^{2} \mathrm{H}_{3}\right]$ nonanoate $(\mathrm{C} 9)$, and [7${ }^{2} \mathrm{H}_{3}$ ] heptanoate (C7), obtained from CDN Isotopes, Pointe Claire, Quebec, Canada). Cells were incubated for 72 hours at $37^{\circ} \mathrm{C}$ in humidified $5 \% \mathrm{CO}_{2} / 95 \%$ air. After the incubation period, the media were collected and the cells were harvested with trypsin-EDTA, washed in PBS, centrifuged at $2500 \mathrm{~g}$ for 5 minutes and resuspended in $300 \mu \mathrm{l}$ water for protein determination. A $100-\mu \mathrm{l}$ sample consisting of two parts incubated media $(67 \mu \mathrm{l})$ and one part cell suspension
(33 $\mu \mathrm{l})$ was prepared and analyzed for acylcarnitines on a Quattro II tandem mass spectrometer (Micromass, Beverly, Massachusetts, USA) as previously described (9). $\left[{ }^{2} \mathrm{H}_{3}\right]$ acylcarnitine concentrations were expressed as the average values $(\mathrm{nmol} / \mathrm{mg}$ protein per 72 hours) from triplicate flasks.

Fibroblasts from 67 patients diagnosed with longchain fatty acid oxidation defects (including the three VLCAD patients described below) were incubated with the $\left[\omega-{ }^{2} \mathrm{H}_{3}\right]$ odd-chain fatty acids listed above. The 67 cell lines represented the following deficiencies: carnitine palmitoyltrasferase I (CPT I), 4 patients; carnitineacylcarnitine translocase (translocase), 5 patients; carnitine palmitoyltransferase II (CPT II), 10 patients; VLCAD, 24 patients (12 cardiomyopathic [VLCAD-C] and 12 rhabdomyolytic/hypoglycemic [VLCAD-M] phenotypes) (8); L-3-hydroxy acyl-CoA dehydrogenase (LCHAD), 19 patients; and mitochondrial trifunctional protein (MTP), 5 patients. The results of these defective cell lines were compared with those obtained from the fibroblasts of 4 normal patients. After 72 hours' incubation, the carnitine esters of the $\left[\omega-{ }^{2} \mathrm{H}_{3}\right]$ fatty acid and $\left[\omega-{ }^{2} \mathrm{H}_{3}\right]$ propionylcarnitine produced during the incubation were measured by tandem mass spectrometry, as described above.

\section{Analytical methods}

Acylcarnitine and organic acid analyses have been described previously (10). The plasma fatty acid metabolites (BHB, AcAc, BHP, BKP, and FFAs) were analyzed by gas chromatography-mass spectrometry $(7,11)$.

\section{Dietary materials}

Portagen formula (containing 30\% of the calories as MCT oil containing trioctanoin and tridecanoin) was provided by Mead Johnson Nutritionals (Evansville, Indiana, USA). Triheptanoin oil was provided by SASOL GmbH (Witten, Germany).

\section{Clinical description}

Three patients with the cardiomyopathic phenotype of VLCAD deficiency were evaluated on diets containing medium-even-chain triglyceride and mediumodd-chain triglyceride (triheptanoin). Their descriptions are as follows.

Patient 1. This 6-year-old Caucasian female presented at 3 days of age with hypothermia and hypoglycemia, which responded to fluid therapy. For the first 3 months, she was regarded as showing failure to thrive with hypotonia and was a poor feeder. Between 3.5 and 7 months of age, she required hospitalization due to respiratory failure, hypertrophic cardiomyopathy with biventricular hypertrophy and pericardial effusion, and hepatomegaly. The diagnosis of VLCAD deficiency was made by blood acylcarnitine analysis on the fourth hospital day, and her diet was immediately changed to Portagen (Mead Johnson Nutritionals) with L-carnitine supplementation. Over the next 12 days, the pericardial effusion and heart size had decreased markedly. She was 
ventilator-dependent and required Nissen fundoplication and gastrostomy for feedings. The precipitating factor was assumed to be tracheitis caused by Staphylococcus aureus. Fibroblast enzyme assay confirmed VLCAD deficiency (activity with palmitoyl-CoA was $0.01 \mathrm{nmol}$ electron-transfer flavoprotein (ETF) reduced $/ \mathrm{min} / \mathrm{mg}$ protein; normal mean/SD $=1.64 / 0.57)(8)$. The patient's medical record provided a molecular diagnostic report that indicated there was a deletion of bp 887-888 in exon 10 along with a splicing mutation preceding exon 18 in the VLCAD gene.

Examinations at 13 and 17 months of age revealed persistent hypertrophic cardiomyopathy with biventricular hypertrophy and compensating hyperdynamic left ventricular function (shortening fraction $45 \%$ ), developmental delay (motor and speech), weakness, and chronic elevation of serum creatine kinase (>1000 IU/l).

At 2 years 7 months and again at 4 years 10 months of age, patient 1 was hospitalized due to infections (urinary tract and bilateral maxillary sinusitis with otitis, respectively). On both occasions, she presented with lethargy, cardiomegaly, rhabdomyolysis, and hypertrophic cardiomyopathy with hepatomegaly. Appropriate antibiotic therapy for the infections coupled with simultaneous infusion of glucose and insulin, for control of lipolysis, expedited her recovery. Follow-up examination 2 months later, at 5 years of age, when she was not acutely ill, continued to demonstrate cardiomyopathy with persistent thickening of the posterior left ventricular wall and interventricular septum. The left ventricular end-diastolic diameter (LVEDD) remained above normal (42; normal for her weight $=30-38$ ) with global hypokinesis (shortening fraction 19\%).

Two months later at age 5 years 2 months, she entered the dietary treatment protocol at Baylor University Medical Center. Initial physical examination revealed hepatomegaly $(8 \mathrm{~cm}$ below the costal margin) and marked weakness (classical Gowers' sign; inability to climb stairs, walk, run, or open doors without assistance).

Patient 2. This 9-year-old Caucasian female presented at 2 days of age with hypoglycemia that responded to fluid therapy. A severe episode occurred at 3 months, with hypoglycemic seizures, vomiting, hypotonia, metabolic acidosis, hepatomegaly, and cardiorespiratory arrest. She had biventricular hypertrophy and pericardial effusion requiring pericardiocentesis to relieve developing tamponade. Following resuscitation she was placed on a low-fat diet with increased carbohydrate. VLCAD deficiency was diagnosed from blood acylcarnitine analysis and later by direct enzyme assay of fibroblasts $(0.37 \mathrm{U} / \mathrm{mg}$ protein; normal $=2.10 \pm 0.31$ $\mathrm{U} / \mathrm{mg}$ protein [mean $\pm \mathrm{SD}]$ ) (12). She had episodes of vomiting, associated with increased levels of serum creatine phosphokinase (CPK) occurring every 3-4 weeks from age 15 months to age 6.5 years, requiring multiple hospitalizations. Between 2.5 and 3.5 years of age, many of the hospitalizations were due to respiratory distress resulting from asthma. She required Flovent (fluticasone propionate; Glaxo Wellcome, Raleigh, North Carolina, USA) and a prednisone liquid preparation frequently for treatment. Intermittent rhabdomyolysis up until the protocol was associated with limited activity due to pain and weakness but no further cardiac involvement or hypoglycemia. During school she had difficulty concentrating on tasks. From age 4 years, she was fed a diet rich in medium-evenchain triglycerides, mainly by gastrostomy.

Patient 3. This 2-year-old Caucasian male weighed 8 lbs. 1 oz. following a normal pregnancy, labor, and cesarean delivery. He became hypoglycemic at 12 hours and was thought to be septic, although cultures were negative. Muscle weakness and hypoglycemia remained prominent clinical features from birth. At 3 months of age, after loss of muscle tone along with increasing feeding problems, he was hospitalized with cardiac and respiratory failure along with hypoglycemia. During that admission, he had hepatomegaly, hypertrophic cardiomyopathy with pericardial effusion (requiring pericardiocentesis), hypotonia, and hypoglycemia. He also required tracheostomy and gastrostomy. Echocardiogram revealed mild left ventricular hypertrophy. Right ventricular dimension in end-diastole was $1.7 \mathrm{~cm}$, interventricular septum in diastole $0.4 \mathrm{~cm}$, interventricular septum in systole $0.8 \mathrm{~cm}$, left ventricular diameter in diastole $3.8 \mathrm{~cm}$, left ventricular dimension in systole 2.2 $\mathrm{cm}$, left posterior wall thickness in diastole $0.5 \mathrm{~cm}$, and left ventricular posterior wall thickness in systole 1.1 $\mathrm{cm}$. The diagnosis of VLCAD deficiency was determined by the blood acylcarnitine profile and plasma FFA profile, which revealed elevated octanoic, decanoic, and tetradecanoic acids. The patient was switched to Portagen formula (Mead Johnson Nutritionals), which resulted in significant improvement. He had two additional hospitalizations, one for gastroenteritis and one due to respiratory syncytial virus, neither of which was associated with congestive heart failure. He recovered from both following fluid therapy. On admission to the oddcarbon diet protocol, he appeared developmentally normal and his speech was precocious. Physical examination excluded hepatomegaly, and his cardiac status was normal. Muscle weakness was demonstrated by his inability to support his own weight when lifted under his arms ("fall through") and his stumbling when running approximately 10-15 feet. He had a Trendelenburg gait when running. The child's medical records revealed that molecular abnormalities had been identified in the VLCAD gene: a 2-bp deletion in exon 8 (bp 842-843).

\section{Results}

In vitro evaluation of oxidation of fatty acid precursors in fibroblasts

Fibroblasts from patient 1 and normal cell lines were evaluated as previously described with C16 (9). In addition, all cell lines were incubated, separately, with $\mathrm{C} 15, \mathrm{C} 9$, and $\mathrm{C} 7$ to evaluate their contribution to 
Table 1

Oxidation of fatty acids by cultured fibroblasts from a patient with VLCAD deficiency

\begin{tabular}{|c|c|c|c|c|c|c|c|c|}
\hline \multicolumn{9}{|c|}{ (Labeled intermediates as acylcarnitines: $\mathrm{nmol} / \mathrm{mg}$ protein $/ 72 \mathrm{~h}$ ) } \\
\hline \multirow[t]{2}{*}{ Palmitate } & $(\mathrm{C} 16)^{\mathrm{A}}$ & $\mathrm{C} 4$ & $\mathrm{C} 6$ & $\mathrm{C} 8$ & $\mathrm{C} 10$ & C12 & C14 & $\mathrm{C} 16$ \\
\hline & Patient & 0.61 & 0.19 & 0.31 & 0.40 & 2.04 & 8.08 & 15.95 \\
\hline \multirow[t]{2}{*}{ Controls $(n=4)$} & Mean & 1.31 & 1.06 & 2.31 & 2.02 & 1.03 & 0.58 & 3.59 \\
\hline & SD & 0.38 & 0.43 & 1.62 & 1.72 & 0.61 & 0.39 & 1.08 \\
\hline \multirow[t]{2}{*}{ Pentadecanoate } & $(\mathrm{C} 15)^{\mathrm{A}}$ & $\mathrm{C} 3$ & $\mathrm{C} 5$ & $\mathrm{C7}$ & $\mathrm{C} 9$ & $\mathrm{C} 11$ & C13 & C15 \\
\hline & Patient & 6.48 & 0.98 & 0.19 & 0.64 & 0.89 & 10.87 & 18.46 \\
\hline \multirow[t]{2}{*}{ Controls $(n=4)$} & Mean & 46.76 & 2.79 & 0.83 & 2.20 & 0.87 & 0.68 & 3.80 \\
\hline & SD & 8.84 & 0.72 & 0.53 & 2.01 & 0.60 & 0.55 & 1.77 \\
\hline \multirow[t]{2}{*}{ Nonanoate } & $(C 9)^{A}$ & $\mathrm{C} 3$ & C5 & $\mathrm{C7}$ & C9 & & & \\
\hline & Patient & 3.77 & 0.72 & 0.22 & 3.58 & & & \\
\hline \multirow[t]{2}{*}{ Controls $(n=4)$} & Mean & 3.90 & 0.44 & 0.26 & 2.00 & & & \\
\hline & SD & 1.47 & 0.19 & 0.10 & 0.63 & & & \\
\hline \multirow[t]{2}{*}{ Heptanoate } & $(C 7)^{A}$ & $\mathrm{C} 3$ & $\mathrm{C} 5$ & $\mathrm{C7}$ & & & & \\
\hline & Patient & 19.78 & 2.62 & 0.32 & & & & \\
\hline \multirow[t]{2}{*}{ Controls $(n=4)$} & Mean & 24.29 & 1.32 & 0.37 & & & & \\
\hline & SD & 5.60 & 0.30 & 0.15 & & & & \\
\hline
\end{tabular}

AData are averages of triplicate incubations of the patient's cell line with each fatty acid.

propionyl-CoA production in vitro. The "chain-length profile" for this patient with VLCAD deficiency is presented in Table 1 . The incubations with $\mathrm{C} 16$ reveal the characteristic profile of acylcarnitines observed in the cardiomyopathic form of this disease - markedly increased C16 and tetradecanoylcarnitine (C14) with an increased ratio of $\mathrm{C} 16 / \mathrm{C} 12(>4.0)(8,13)$. This patient's cells also demonstrated increased levels of $\mathrm{C} 15$ when incubated with pentadecanoate. The relative amounts (nmol/mg protein/72 hours) of propionylcarnitine (C3) produced after 72 hours' incubation with the odd-carbon fatty acid precursors (C15, $\mathrm{C}$, and $\mathrm{C7}$ ) revealed that the production of $\mathrm{C} 3$ from C15 was greatly reduced (6.48) compared with normal cells (46.76). The amount derived from C9, although normal (3.77), was also unimpressive. The amount derived from C7 (19.78) was equivalent to that observed with normal cell lines (24.29), which was three to five times greater than that observed with either $\mathrm{C} 15$ or $\mathrm{C} 9$. The relative contributions of these odd-carbon fatty acids to production of propionylCoA are presented in Figure 1.

Clinical study with triheptanoin versus MCT oil in the diet

The investigative protocol (Baylor IRB no. 099-135; FDA IND no. 59,303) was designed, with the informed consent of the patients' parents, to compare the recommended dietary therapy (MCT oil containing trioctanoin and tridecanoin) with a diet containing the same amount of triheptanoin (C7 triglyceride). We evaluated the two diets as they would be delivered at home rather than as a bolus of the individual oils. The protocol involved an initial admission for 9 days with follow-up visits at 2, 6, 12, and 18 months. These three patients were receiving diets delivered by gastrostomy that included Portagen formula (Mead Johnson Nutritionals) containing $30 \%$ of the calories as MCT. During the Portagen diet, samples for metabolite profiling were taken during and following a morning meal on the third day of admission. The patients were then given a diet in which triheptanoin was substituted, isocalorically, for MCT. Samples for metabolite profiles were taken during and after breakfast on the sixth day of admission to ensure that the profile reflected the triheptanoin diet. Table 2 presents the analyses of the different metabolites in plasma under the two dietary regimens for patient 1 as an example of the three patients. This investigation included quantification of both acylcarnitines and free acids in plasma during the meals. The MCT "breakfast" was associated with a decrease in the most specific acylcarnitine that accumulates in VLCAD deficiency, cis-5-C14:1 (C14:1) derived from

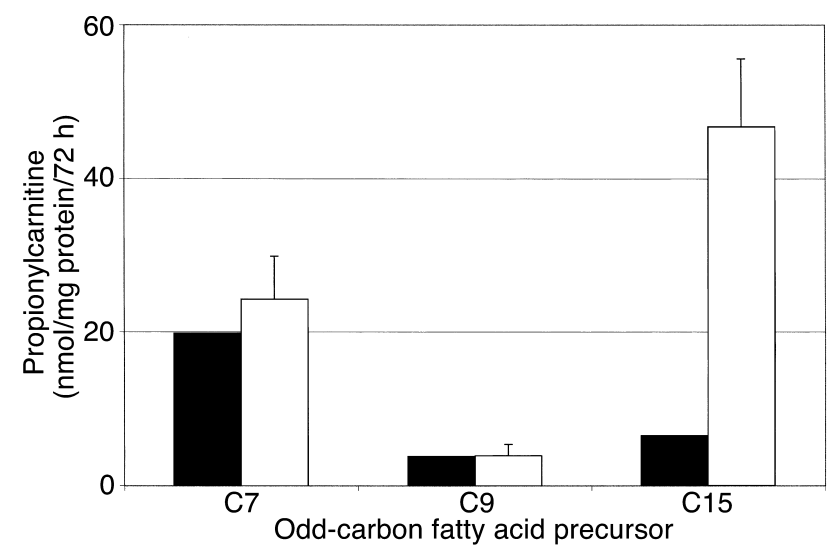

Figure 1

Production of $\left[3-{ }^{2} \mathrm{H}_{3}\right]$ propionylcarnitine from $\mathrm{C} 7, \mathrm{C} 9$, and $\mathrm{C} 15$ by cultured fibroblasts from the VLCAD patient (black bars) and 50 control subjects (white bars, mean $\pm \mathrm{SE}$ ). 
Table 2

Plasma metabolite levels $(\mu \mathrm{M})$ during meals with MCT and triheptanoin

\begin{tabular}{|c|c|c|c|c|c|c|c|c|c|}
\hline \multicolumn{10}{|c|}{ MCT $\operatorname{diet}^{\mathrm{A}}$} \\
\hline Time (min) & $\mathrm{C} 8$ & $\mathrm{BHB}$ & AcAc & $\mathrm{BHP}$ & BKP & $\mathrm{C} 8 \mathrm{AC}^{\mathrm{B}}$ & $\mathrm{C} 5 \mathrm{AC}$ & $\mathrm{C} 3 \mathrm{AC}$ & C14:1 \\
\hline 0 & 51 & 35 & 18 & 0 & 0 & 0.0 & 0.1 & 0.4 & 1.3 \\
\hline 30 & 1518 & 993 & 343 & 0 & 0 & 0.1 & 0.1 & 0.4 & 1.1 \\
\hline 104 & 618 & 1600 & 429 & 0 & 0 & 0.2 & 0.1 & 0.4 & 0.4 \\
\hline 143 & 289 & 1074 & 254 & 0 & 0 & 0.1 & 0.1 & 0.3 & 0.3 \\
\hline 168 & 263 & 827 & 155 & 0 & 0 & 0.1 & 0.1 & 0.3 & 0.2 \\
\hline \multicolumn{10}{|c|}{ Triheptanoin $\operatorname{diet}^{\mathrm{A}}$} \\
\hline Time (min) & $\mathrm{C7}$ & $\mathrm{BHB}$ & $A c A c$ & $\mathrm{BHP}$ & $\mathrm{BKP}$ & $\mathrm{C} 7 \mathrm{AC}^{\mathrm{B}}$ & C5 AC & $\mathrm{C} 3 \mathrm{AC}$ & C14:1 \\
\hline 0 & 11 & 13 & 8 & 0 & 0 & 0.0 & 0.1 & 0.8 & 0.5 \\
\hline 30 & 768 & 254 & 48 & 0 & 0 & 0.1 & 0.5 & 1.2 & 0.5 \\
\hline 60 & 565 & 211 & 62 & 16.3 & 8.1 & 0.1 & 0.7 & 2.5 & 0.5 \\
\hline 90 & 1493 & 328 & 79 & 51.0 & 18.6 & 0.2 & 1.1 & 3.8 & 0.4 \\
\hline 120 & 312 & 270 & 84 & 68.5 & 17.2 & 0.2 & 1.1 & 4.0 & 0.3 \\
\hline 150 & 81 & 24 & 16 & 11.4 & 3.3 & 0.1 & 0.4 & 2.8 & 0.4 \\
\hline 180 & 110 & 25 & 8 & 5.9 & 1.7 & 0.1 & 0.4 & 2.1 & 0.2 \\
\hline Limit $(\mu \mathrm{M})$ & & & & & & $<0.06$ & 0.37 & 2.64 & 0.18 \\
\hline
\end{tabular}

${ }^{A}$ Equivalent oil composition in the diet $=3.7 \mathrm{~g} / \mathrm{kg} / 24 \mathrm{~h} .{ }^{\mathrm{B}} \mathrm{AC}$, acylcarnitine. Upper limit for $\mathrm{C} 8 \mathrm{AC}=0.23$; upper limit for $\mathrm{C} 7 \mathrm{AC}<0.06 \mu \mathrm{M}$.

oleate, but no other perturbation of plasma acylcarnitines was observed. However, there was substantial production of $\mathrm{BHB}$ and $\mathrm{AcAc} 60-120$ minutes into the meal. The level of $\mathrm{C} 3$ was not affected.

During the breakfast containing isocaloric amounts of triheptanoin, there were substantial increases in normal plasma ketone bodies (BHB and AcAc) as well as in the odd-carbon ketone bodies (BKP and BHP) (peak plasma levels at 90-150 minutes). Associated with these elevations was the simultaneous increase in C3. The plasma levels of C14:1 were already reduced prior to this meal test, possibly because the patients had been receiving the triheptanoin formula for more than 48 hours before the actual meal test.

Analysis of urinary organic acids revealed excretion of both 4 and 5 carbon ketones (BHB, AcAc, BHP, and BKP), FFAs (C7 and octanoate [C8]), and equivalent amounts of dicarboxylates (suberate-C8 or
pimelate-C7) derived by $\omega$-oxidation of the dietary fatty acids (Table 3). Excretion of BHB and AcAc during the triheptanoin diet, with all three patients, was consistently lower than that observed during the even-chain MCT diet.

\section{Indices of propionate overload}

One of the main concerns regarding the use of odd-carbon fatty acids has been the theoretical possibility of creating metabolic derangements similar to those observed in patients with propionic acidemia. In fact, the excretions of indices of propionyl overload (3-hydroxypropionate, propionylglycine, and methylcitrate) in ten urine samples from patient 1 (collected over 12 months) were orders of magnitude lower than in samples from 12 propionic acidemia patients (Figure 2). This was true in all three patients. Furthermore, propionylglycine was never detected in urine samples

Table 3

Urine metabolites: meals with MCT and triheptanoin ( $\mathrm{mmol} / \mathrm{mol}$ creatinine)

\begin{tabular}{|c|c|c|c|c|c|c|}
\hline \multicolumn{7}{|c|}{ MCT oil } \\
\hline Time (min) & $\mathrm{BHB}$ & AcAc & $\mathrm{BHP}$ & Octanoic ${ }^{A}$ & Suberic & Pimelic \\
\hline Before & 13 & 7 & 0 & 17 & 62 & 9 \\
\hline 28 & 31 & 24 & 0 & 39 & 31 & 5 \\
\hline 88 & 636 & 547 & 5 & 293 & 158 & 10 \\
\hline \multicolumn{7}{|c|}{ Triheptanoin oil } \\
\hline Time (min) & $\mathrm{BHB}$ & AcAc & BHP & Heptanoic & Suberic & Pimelic \\
\hline 30 & 3 & 0 & 0 & 10 & 3 & 26 \\
\hline 60 & 184 & 85 & 144 & 180 & 25 & 136 \\
\hline 140 & 221 & 195 & 164 & 202 & 52 & 223 \\
\hline 160 & 9 & 0 & 11 & 92 & 39 & 253 \\
\hline Upper limit & 22 & 7 & $<5$ & 0 & 9 & 10 \\
\hline
\end{tabular}

AUpper limit for octanoate $=3 \mathrm{mmol} / \mathrm{mol}$ creatinine. 


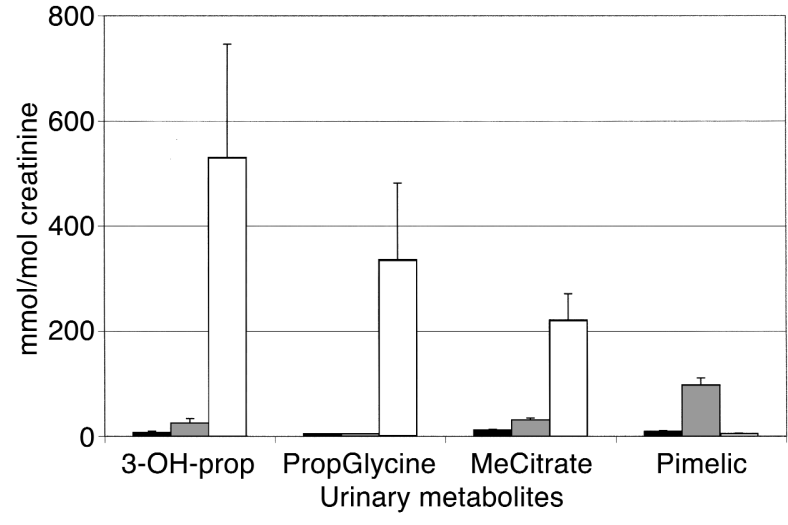

Figure 2

Urinary excretion of indices of propionyl overload (3-hydroxypropionate [3-OH-prop], propionylglycine [PropGlycine], methylcitrate [MeCitrate]) and pimelic in urine samples from 50 normal subjects (black bars), the VLCAD patients (gray bars; ten samples collected over 12 months), and ten patients with propionic acidemia (white bars).

from any of the patients on the triheptanoin diet, while it is often excreted in large amounts in patients with propionic acidemia. Thus, there was no sign of propionate overload in VLCAD patients treated for up to 27 months with the triheptanoin diet.

\section{Clinical course following} triheptanoin dietary therapy

A summary of the primary clinical features of the three VLCAD patients is presented in Table 4 . The diagnosis was determined in all three patients at 3 months of age when each developed hypertrophic cardiomyopathy. The clinical and biochemical details of each patient, following initiation of the triheptanoin diet, are described as follows.

Patient 1. This 6-year-old female with VLCAD deficiency had endured frequent recurrent rhabdomyolysis and marked weakness with exercise intolerance since her severe cardiomyopathic episode at 3.5 months of age. On admission at age 5 years 2 months (February 2000 ), she was unable to stand from a sitting position (Gowers' sign) and could not climb stairs or open doors without assistance. Within 5 hours of her first triheptanoin meal, she was able to stand up without assistance and open heavy doors for her parents. Her level of activity increased remarkably and quickly. Both parents were amazed by the sudden improvement in muscle strength that occurred within the first 12 hours of dietary triheptanoin. The hepatomegaly $(8 \mathrm{~cm})$ was undetectable after 6 days of therapy (at discharge). There was an 8-lb. weight gain during the 9-day admission that was subsequently controlled by reduction of carbohydrate in her diet. Her echocardiogram 2 weeks prior to admission indicated a shortening fraction of only $19 \%$ (normal $>30 \%$ ) and a LVEDD of 4.2 with reduced function. Mild concentric left ventricular hypertrophy and global hypokinesis were also noted at that time. Repeat echocardiogram 1 month later demonstrated a shortening fraction of $30 \%$ (ejection fraction of 57\%). Mild thickness of the interventricular septum (in diastole, $5.9 \mathrm{~mm}$; in systole, $9.8 \mathrm{~mm}$ ) and the posterior wall of the left ventricle (left ventricle in diastole, $43.9 \mathrm{~mm}$; in systole, $30.9 \mathrm{~mm}$ ) was noted. Her clinical improvement without any limitation in normal activity made a planned institution of digoxin therapy unnecessary. Patient 1 has been followed for 26 months on therapy with dietary triheptanoin at a dose of $3.5-4.0 \mathrm{~g} / \mathrm{kg} / \mathrm{d}$. She has had no serious episodes of rhabdomyolysis or cardiac complications. She is now regarded as a normal 6-year old, is doing well in school, and exercises on a trampoline.

Patient 2. During the initial admission at age 7 years 11 months (June 2000), patient 2's physical examination revealed a weight of $30 \mathrm{~kg}$, mild hepatomegaly $(2 \mathrm{~cm})$, abnormal extraocular movements (inability to elevate her right eye), decreased response to light in the right eye, disparity of circumference and length of her legs, shoe size disparity, partial Gowers' sign, and decreased deep-tendon reflexes. She complained of leg pain following exercise testing and generally preferred to rest in bed. CPK on admission was $2400 \mathrm{IU} / 1$, and the plasma total carnitine level was $16 \mu \mathrm{M}$. She continued her MCT-containing $(3.3 \mathrm{~g} / \mathrm{kg} / \mathrm{d})$ diet and was begun on carnitine supplement (500 mg four times daily) 1 day prior to the morning meal (MCT) test. There was no apparent physical or chemical improvement while on the MCT diet. Triheptanoin $(3.2 \mathrm{~g} / \mathrm{kg} / \mathrm{d})$ was substituted for MCT in her diet on the second day. Within 24 hours, she had become very active (and mildly hypomanic) without any limitations, muscle pain, or Gowers' sign. The morning meal test containing triheptanoin was done on the fifth day ( 3 days after starting this diet). At discharge, she continued to be active and no longer had a partial Gowers' sign, her liver size was normal, and the ptosis and decreased reaction to light

\section{Table 4}

Clinical features of VLCAD patients on triglyceride diets

\begin{tabular}{|c|c|c|c|}
\hline & Patient 1 & Patient 2 & Patient 3 \\
\hline Diagnosis (age) & $3.5 \mathrm{mos}$ & $3 \mathrm{mos}$ & $3 \mathrm{mos}$ \\
\hline $\begin{array}{l}\text { Cardiomyopathy } \\
\text { (age of onset) }\end{array}$ & $3.5 \mathrm{mos}$ & $3 \mathrm{mos}$ & $3 \mathrm{mos}$ \\
\hline $\begin{array}{l}\text { MCT diet } \\
\text { (age initiated) }\end{array}$ & $3.5 \mathrm{mos}$ & $4 \mathrm{yrs}$ & $3 \mathrm{mos}$ \\
\hline Months on diet & 58 & 60 & 21 \\
\hline Clinical features: & $\begin{array}{c}\text { Hypoglycemia } \\
\text { Cardiomyopathy } \\
\text { Weakness } \\
\text { Hepatomegaly } \\
\text { Muscle pain }\end{array}$ & $\begin{array}{l}\text { Hypoglycemia } \\
\text { Weakness } \\
\text { Hepatomegaly } \\
\text { Muscle pain }\end{array}$ & $\begin{array}{c}\text { Hypoglycemia } \\
\text { Weakness } \\
\text { Muscle pain }\end{array}$ \\
\hline $\begin{array}{l}\text { Triheptanoin diet } \\
\text { (age initiated) }\end{array}$ & 5 yrs & $8 \mathrm{yrs}$ & $2 \mathrm{yrs}$ \\
\hline Months on diet & 27 & 23 & 4 \\
\hline Clinical features ${ }^{A}$ & $\begin{array}{l}\text { Muscle pain } \\
\text { (rarely) }\end{array}$ & $\begin{array}{l}\text { Muscle pain } \\
\text { (occasionally) }\end{array}$ & None \\
\hline
\end{tabular}

AExcept for cardiomyopathy, all resolved during initial admission. Echocardiogram was normal in patient 1 at 30 days. 
Table 5

Acylcarnitines produced from $\left[\omega-{ }^{2} \mathrm{H}_{3}\right]$ odd-chain fats by fibroblasts with $\beta$-oxidation defects

\begin{tabular}{|c|c|c|c|c|c|c|c|}
\hline \multicolumn{2}{|c|}{ Fatty acid precursor } & \multicolumn{2}{|c|}{ C7 } & \multicolumn{2}{|c|}{$\mathrm{C} 9$} & \multicolumn{2}{|c|}{$\mathrm{C} 15$} \\
\hline Disorder & Ester & {$\left[7-{ }^{2} \mathrm{H}_{3}\right] \mathrm{C} 7^{\mathrm{A}}$} & {$\left[3-{ }^{2} \mathrm{H}_{3}\right] \mathrm{C} 3$} & {$\left[9-{ }^{2} \mathrm{H}_{3}\right] \mathrm{C} 9$} & {$\left[3-{ }^{2} \mathrm{H}_{3}\right] \mathrm{C} 3$} & {$\left[15-{ }^{2} \mathrm{H}_{3}\right] \mathrm{C} 15$} & {$\left[3-{ }^{2} \mathrm{H}_{3}\right] \mathrm{C} 3$} \\
\hline CPT I & Mean & 0.48 & 29.15 & 2.33 & 5.26 & 0.10 & 10.59 \\
\hline \multirow[t]{2}{*}{$(n=4)$} & SD & 0.26 & 14.88 & 1.56 & 4.23 & 0.12 & 7.94 \\
\hline & Range & $0.2-0.6$ & $15.4-45.0$ & $1.2-4.6$ & $1.9-11.3$ & $0-0.2$ & $3.8-20.9$ \\
\hline Translocase & Mean & 0.91 & 13.62 & 18.46 & 1.04 & 37.27 & 8.18 \\
\hline \multirow[t]{2}{*}{$(n=5)^{\mathrm{B}}$} & SD & 0.33 & 14.28 & 10.95 & 1.82 & 14.77 & 12.54 \\
\hline & Range & $0.5-1.3$ & $2.8-37.0$ & $3.8-33.0$ & $0-4.3$ & $15.7-52.8$ & $0.8-29.6$ \\
\hline CPT II & Mean & 0.68 & 19.97 & 15.65 & 3.51 & 42.08 & 13.39 \\
\hline \multirow[t]{2}{*}{$(n=10)^{\mathrm{B}}$} & SD & 0.25 & 14.80 & 6.85 & 3.17 & 16.94 & 12.81 \\
\hline & Range & $0.3-1.0$ & $4.3-42.4$ & $7.9-26.5$ & $0-8.3$ & $17.5-67.1$ & $0-43.2$ \\
\hline VLCAD-C & Mean & 0.33 & 35.94 & 3.27 & 8.21 & 22.38 & 13.47 \\
\hline \multirow[t]{2}{*}{$(n=12)$} & SD & 0.16 & 22.85 & 1.89 & 4.47 & 7.88 & 5.85 \\
\hline & Range & $0.1-0.5$ & $6.6-90.7$ & $1.2-8.0$ & $3.0-18.2$ & $11.9-35.5$ & $6.5-26.1$ \\
\hline VLCAD-M & Mean & 0.47 & 39.68 & 2.51 & 7.43 & 9.51 & 39.99 \\
\hline \multirow[t]{2}{*}{$(n=12)$} & SD & 0.22 & 29.41 & 1.01 & 5.16 & 4.45 & 26.39 \\
\hline & Range & $0.2-0.8$ & $8.7-103.7$ & $0.9-4.6$ & 2.9-20.2 & $2.9-16.2$ & $16.9-111.2$ \\
\hline LCHAD & Mean & 0.51 & 31.69 & 4.24 & 6.32 & 11.49 & 15.58 \\
\hline \multirow[t]{2}{*}{$(n=19)$} & SD & 0.24 & 16.04 & 3.70 & 4.51 & 4.71 & 7.51 \\
\hline & Range & $0.2-1.0$ & $6.6-59.9$ & $1.5-18.2$ & $0.9-18.9$ & $4.0-20.5$ & $3.5-34.6$ \\
\hline MTP & Mean & 0.76 & 40.75 & 2.50 & 5.57 & 12.83 & 18.20 \\
\hline \multirow[t]{2}{*}{$(n=5)$} & SD & 0.64 & 24.90 & 0.52 & 2.23 & 7.35 & 11.47 \\
\hline & Range & $0.3-1.9$ & $18.1-81.5$ & $1.9-3.2$ & $3.1-9.0$ & $3.4-20.4$ & $7.1-30.7$ \\
\hline Controls & Mean & 0.37 & 24.29 & 2.00 & 3.90 & 3.80 & 46.76 \\
\hline$(n=4)$ & SD & 0.15 & 5.60 & 0.63 & 1.47 & 1.77 & 8.84 \\
\hline
\end{tabular}

${ }^{A}$ Deuterated acylcarnitines. ${ }^{B}$ Data is influenced by a mixture of distinct clinical phenotypes affecting SD: translocase (2 phenotypes), CPT II (3 phenotypes).

by the right eye had disappeared. She had gained $4 \mathrm{lbs}$. during the 9-day admission. Reduction of the dietary carbohydrate corrected the weight gain problem.

During the next 3 months, patient 2 was briefly hospitalized three times for fluid therapy due to increased plasma CPK levels. During this interval, she had no further signs of asthma and her chronic medications (including Flovent, $110 \mathrm{mcg}$, two puffs twice daily) were discontinued. During the next 15 months, she had no hospitalizations. Her "attention deficit" at school was no longer an issue.

Recent, prophylactic reintroduction of Flovent, in the absence of symptoms, was associated with recurrence of muscle pain and increased plasma CPK levels (up to 50,000 IU/l) requiring several hospitalizations. With gradual removal of this medication, the plasma CPK level had decreased to $284 \mathrm{IU} / 1$. This patient has been on the diet for 22 months.

Patient 3. Patient 3's upper body strength was of great concern before the study. He had always been in physical therapy. At the time of discharge, he appeared to have normal activity for a 2-year-old. He was able to suspend his weight under the arms for more than 10 seconds and could run more than 100 yards without stumbling. Attempts at formal muscle testing revealed significantly improved strength after running without signs of fatigue. His plasma free carnitine had risen from $9 \mu \mathrm{M}$ on the day of admission to $38 \mu \mathrm{M}$ at discharge with supplementation.
Patient 3 had gained $8.5 \mathrm{lbs}$. over 2 months on the triheptanoin diet $(2.6 \mathrm{~g} / \mathrm{kg} / \mathrm{d})$, presumably due to continued high carbohydrate supplementation at home. The family elected to discontinue the triheptanoin diet trial before reduction of excessive carbohydrate could be implemented. Following his return to the MCT-containing diet, episodes of rhabdomyolysis with hospitalizations resumed, with plasma CPK levels up to $60,000 \mathrm{IU} / 1$.

In vitro studies of fibroblasts from patients with long-chain fat oxidation disorders

Sixty-seven cell lines from patients with long-chain fat oxidation disorders were tested with the $\omega$-deuterated odd-chain fatty acids C15, C9, and C7, in culture (Table 5). These cell lines represent the following deficiencies: CPT I, 4 patients; translocase, 5 patients; CPT II, 10 patients; VLCAD, 24 patients (12 cardiomyopathic [VLCAD-C] and 12 rhabdomyolytic/hypoglycemic [VLCAD-M] phenotypes) (8); LCHAD, 19 patients; and MTP, 5 patients. The quantities ( $\mathrm{nmol} / \mathrm{mg}$ protein $/ 72$ hours) of carnitine esters of the $\omega^{-2} \mathrm{H}_{3}$-fatty acids $\left(\left[15-{ }^{2} \mathrm{H}_{3}\right] \mathrm{C} 15,\left[9-{ }^{2} \mathrm{H}_{3}\right] \mathrm{C} 9\right.$, and $\left.\left[7-{ }^{2} \mathrm{H}_{3}\right] \mathrm{C} 7\right)$ were compared with those of $\left[3-{ }^{2} \mathrm{H}_{3}\right]$ propionylcarnitine produced from each during the 72-hour incubation period and those observed with the control cell lines.

Markedly increased quantities of the precursor fatty acid as a carnitine ester generally reflect a requirement for the defective enzyme for its uninhibited oxidation. 
This is illustrated by the levels of $\left[15-{ }^{2} \mathrm{H}_{3}\right]$ pentadecanoyl-carnitine with all of the defects except that of CPT I cells, which cannot produce long-chain acylcarnitines effectively. The amounts of C9 were distinctly abnormal with translocase- and CPT II-deficient cells, suggesting a significant dependency on these enzymes for its oxidation. There was little, if any, accumulation of $\left[7-{ }^{2} \mathrm{H}_{3}\right]$ heptanoyl-carnitine with any of the cell lines, suggesting effective oxidation in all cell lines with longchain fat oxidative defects.

Very small amounts of $\left[3-{ }^{2} \mathrm{H}_{3}\right]$ propionylcarnitine were produced from $\left[9-{ }^{2} \mathrm{H}_{3}\right]$ nonanoate compared with the other precursors even with normal cell lines. $\left[9_{-2}{ }^{2} H_{3}\right]$ nonanoate proved to be the least desirable as a potential dietary nutrient for any of the disorders.

Overall, the amounts of $\left[3-{ }^{2} \mathrm{H}_{3}\right]$ propionylcarnitine produced from $\mathrm{C} 7$ were comparable to or greater than those amounts produced by normal cell lines or the defective cell lines incubated with $\mathrm{C} 15$. These results focused attention on $\mathrm{C} 7$, in the form of triheptanoin, as a potential dietary component for treatment of all long-chain mitochondrial fat oxidation disorders.

\section{Discussion}

Current treatment of long-chain fat oxidation disorders. The plasma membrane carnitine transporter defect is the only one of the mitochondrial fat oxidation disorders for which an effective treatment (carnitine supplementation) is essentially a cure, since mitochondrial $\beta$-oxidation is intact. Current treatment for the other long-chain fat oxidation disorders, including VLCAD deficiency, is designed to (a) reduce the amount of long-chain dietary fat while covering the needs for essential fatty acids, (b) provide carnitine to convert potentially toxic long-chain acyl-CoAs to acylcarnitines, (c) provide a large fraction of the calories as carbohydrates (including cornstarch at night) to reduce body fat utilization and prevent hypoglycemia, and (d) provide about one-third of the calories as even-chain MCT. The recommended use of even-carbon MCT for treatment of these disorders is based on the notion that the metabolism of the eight to ten carbon fatty acids in MCT oil is independent of CPT I, translocase, CPT II, VLCAD, trifunctional Protein, and LCHAD enzyme activities. It has been assumed that the even-carbon medium-chain fatty acids simply enter the mitochondrion as carboxylates before activation and, therefore, require only those $\beta$-oxidative enzymes with shorter chain-length specificity for further oxidation and energy production. A significant advantage of MCT dietary therapy for long-chain fat oxidation disorders is their conversion to C4 ketone bodies by the liver for export as an energy source for other organ systems, including the brain. However, although MCT oil is a good source of calories for patients with long-chain fat oxidation disorders, it does not meet the needs of those patients prone to recurrent hypoglycemia. The evencarbon fatty acids in MCT (octanoate and decanoate), like all even-carbon fatty acids, are not gluconeogenic. Although the glycerol moiety of MCT oil is gluconeogenic, the amount of glucose it can generate is very small.

It is well recognized that fasting, often due to infection, results in profound hypoglycemia and massive lipid mobilization that lead to severe symptoms such as cardiomyopathy, rhabdomyolysis, and sudden death (14). Avoidance of fasting, frequent feeding (increased carbohydrate), and supplemental bedtime cornstarch (originally introduced for therapy of type I glycogenosis) (15) are all recommended to prevent lipolysis. The use of MCT in the diet has been reported to alleviate acute cardiomyopathy with pericardial effusion in acutely ill VLCAD patients with significant, but not always acceptable, recovery, as seen in our patient (16, 17). Currently, during an acute infection or illness secondary to fasting, the only recourse is intravenous glucose, often with insulin infusion, to control the associated stimulation of lipolysis.

Patient studies. Before any clinical intervention, fibroblasts from the three patients were cultured with $\left[\omega-{ }^{2} \mathrm{H}_{3}\right]$ fatty acids (even- and odd-chain) to determine which, if any, fatty acids might be used in the diet (Table 1). Incubations with $\left[\omega^{-2} \mathrm{H}_{3}\right]$ pentadecanoate or $\left[\omega-{ }^{2} \mathrm{H}_{3}\right]$ palmitate resulted in similar accumulations of pentadecanoyl- and palmitoyl-carnitine. This indicated that pentadecanoyl-CoA, like palmitoyl-CoA, is a substrate for the defective VLCAD enzyme. Incubation of $\left[\omega^{-2} \mathrm{H}_{3}\right]$ odd-carbon substrates with fibroblasts always results in a much greater accumulation of $\left[\omega-{ }^{2} \mathrm{H}_{3}\right]$ propionylcarnitine than is seen in blood acylcarnitine profiles $(<2.64$ $\mu \mathrm{M})$. The slow utilization of propionyl-CoA reflected by the accumulation of $\mathrm{C} 3$ is not understood. However, it allows comparison of the relative production of propionyl-CoA, in vitro, from different odd-carbon substrates. One possibility is that the concentrations of other anaplerotic substrates (glucose, glutamate, etc.) are so high in the culture medium that entry of propionyl groups into the CAC is impaired.

In normal cells, $\left[\omega-{ }^{2} \mathrm{H}_{3}\right]$ pentadecanoate $(\mathrm{C} 15)$ is associated with the greatest amount of $\left[\omega-{ }^{2} \mathrm{H}_{3}\right]$ propionylcarnitine. However, in VLCAD-deficient cells, the amount of $\left[\omega^{-2} \mathrm{H}_{3}\right]$ pentadecanoyl-carnitine that accumulates suggests that it is not suitable as a potential dietary substrate. The amount of $\left[\omega-{ }^{2} \mathrm{H}_{3}\right]$ propionylcarnitine produced from $\left[\omega^{2} \mathrm{H}_{3}\right]$ heptanoate is also high and much greater than that produced from $\left[\omega-{ }^{2} \mathrm{H}_{3}\right]$ nonanoate (C9). This relationship is extremely reproducible. The VLCAD-deficient cells produced much less $\left[\omega-{ }^{2} \mathrm{H}_{3}\right]$ propionylcarnitine than did normal cells when incubated with $\left[\omega-{ }^{2} \mathrm{H}_{3}\right]$ pentadecanoate. In the absence of the VLCAD enzyme in the mitochondria, it is possible that only the smaller peroxisomal contribution to the oxidation of $\left[\omega-{ }^{2} \mathrm{H}_{3}\right]$ pentadecanoate can be observed (18). The results with $\left[\omega-{ }^{2} \mathrm{H}_{3}\right]$ heptanoate (C7) suggested that heptanoate (as a triglyceride) was the obvious candidate for dietary 
therapy because (a) normal amounts of $\left[\omega-{ }^{2} \mathrm{H}_{3}\right]$ propionylcarnitine were produced by the VLCAD-deficient cells and (b) there was no significant accumulation of $\left[\omega-{ }^{2} \mathrm{H}_{3}\right]$ heptanoyl-carnitine.

The results of the chain-length panel incubations clearly demonstrated that $\left[\omega-{ }^{2} \mathrm{H}_{3}\right]$ heptanoate was converted to $\left[\omega-{ }^{2} \mathrm{H}_{3}\right]$ pentanoyl-CoA and then to $\left[\omega-{ }^{2} \mathrm{H}_{3}\right]$ propionyl-CoA in this VLCAD cell line. The activation of heptanoate is probably catalyzed by the medium-chain acyl-CoA synthetase. The initial oxidation of heptanoyl-CoA is most likely catalyzed by medium-chain acyl-CoA dehydrogenase. $\left[\omega-{ }^{2} \mathrm{H}_{3}\right]$ pentanoyl-CoA is oxidized to 3 -keto- $\left[\omega-{ }^{2} \mathrm{H}_{3}\right]$ pentanoyl$\mathrm{CoA}$, which is cleaved to acetyl-CoA and $\left[\omega-{ }^{2} \mathrm{H}_{3}\right]$ propionyl-CoA by the short-chain 3-ketoacyl-CoA thiolase (acetoacetyl-CoA thiolase). This is evidenced by the production and accumulation of $\left[\omega-{ }^{2} \mathrm{H}_{3}\right]$ propionylcarnitine in vitro.

Further evidence for the above was provided by the results of in vivo testing of patient 1 (as well as patients 2 and 3) with meals containing MCT oil or triheptanoin (Tables 2 and 3). During the meal with MCT oil, the plasma and urine metabolite profiles were consistent with previous experiences in that there was a rapid appearance of the four-carbon ketone bodies (BHB and AcAc). The five-carbon ketone bodies (BHP and $\mathrm{BKP}$ ) were not detected in plasma, and there was no increment in C3. As expected, both octanoate and suberate were excreted in the urine. During the meal with triheptanoin, there was a rapid appearance of both the four-carbon and the five-carbon ketone bodies. The latter reached peak levels in 90-120 minutes with a simultaneous peak of $\mathrm{C} 3$. There were no significant increases in heptanoyl- or pentanoyl-carnitine. Plasma heptanoate achieved a peak level similar to that of plasma octanoate with the MCT oil meal. The urine studies reflected the expected increased excretion of BHB, AcAc, BHP, heptanoate, and pimelate, each derived from triheptanoin. These results confirm the integrity of the proposed pathway described from patient 1's fibroblasts.

There was no change in these patients' clinical status during the days they received MCT oil. However, within 5-12 hours of the first meal containing triheptanoin, there was an almost immediate improvement in muscle strength, endurance, and activity. The increased performance, resolution of hepatomegaly, and cardiomyopathy in patient 1 has persisted during the 26 months of her trial without any major episodes of rhabdomyolysis. A similar improvement (15 months without any hospitalizations) was experienced by patient 2 until the reintroduction of steroid-containing medications. Patient 3 also experienced significant improvement in muscle strength and endurance during the 4-month trial of dietary triheptanoin. The return to conventional therapy was associated with renewed episodes of rhabdomyolysis. These dramatic and persistent improvements support the notion that the primary problem in VLCAD deficiency and other long-chain fat oxidation disorders is the severe energy deficit leading to chronic and acute cardiomyopathy (19) and recurrent rhabdomyolysis.

There has been no evidence for any chemical toxicity from the triheptanoin diet during the 26-month treatment interval involving these three patients. Serial studies of blood chemistries (including acylcarnitines) and urinary organic acid analysis have shown no significant abnormalities. The theoretical early concerns of possible propionate overload and "iatrogenic propionic acidemia" were soon eliminated, as illustrated in Figure 2. The levels of 3-hydroxypropionate, propionylglycine, and methylcitrate were orders of magnitude lower than those observed with serial samples from patients with propionic acidemia. Surprisingly, propionylglycine was not detected in any of the samples from the patients. This suggests that the propionyl-CoA pool does not increase sufficiently in hepatocytes oxidizing heptanoate to form this glycine conjugate. (The glycine- $N$-acylase was intact in the patients as evidenced by hippurate [benzoylglycine] excretion.) It also suggests that much of the oxidized heptanoate is converted to the five-carbon ketone bodies (BHP and BKP) in the liver.

Each of these patients experienced rapid weight gain, initially. Since patients with VLCAD deficiency are frequently placed on a low-fat high-carbohydrate diet, the excess carbohydrate while receiving triheptanoin appears to be responsible for the weight gain. When the carbohydrate content is reduced to more normal levels, the weight gain is actually curtailed and actual weight loss can occur in the absence of any symptoms (patients 1 and 2). There has been no weight problem with subsequent patients in this dietary protocol. The triheptanoin diet is initiated with normal, not augmented, amounts of carbohydrate.

Why is tribeptanoin more effective than trioctanoin for treatment of long-chain fat oxidation disorders? The remarkable improvement in the clinical and biochemical condition of patient 1 with VLCAD deficiency occurred almost immediately, when her dietary treatment with MCT oil was changed to treatment with triheptanoin. Given the similarity of structure of octanoate and heptanoate, one has to consider differences in their metabolism and metabolic effects.

The metabolism of octanoate has been investigated much more thoroughly than that of heptanoate. This is because very small amounts of odd-chain fatty acids are present in most human diets. However, there is an obvious difference in the metabolism of the two compounds: the catabolism of octanoate leads only to acetyl-CoA, while the catabolism of heptanoate leads to acetyl-CoA and propionyl-CoA. The provision of propionyl-CoA has two important metabolic consequences: (a) propionyl-CoA is an anaplerotic substrate for the CAC in all tissues, and (b) it is gluconeogenic in liver and kidney cortex. Increasing the supply of propionyl-CoA or of other anaplerotic precursors may have little impact on the 
biochemical and physiological homeostasis, as well as on the maximum exercise capacity, of a healthy subject $(20,21)$. However, it could be crucial in patients with VLCAD deficiency and other longchain fat oxidation disorders. Indeed, patients with these defects have a clear decrease in their ability to generate energy in their muscle and/or heart (chronic muscle weakness, cardiomyopathy, rhabdomyolysis). This occurs despite a high-carbohydrate, low-fat diet supplemented with MCT oil, which provides ample acetyl-CoA to run the CAC in their tissues. One might postulate the need to have some of the acetyl-CoA be derived from fatty acid oxidation.

However, healthy subjects who are on a high-carbohydrate, fat-free diet (Ornish or Pritikin diet) have no muscle weakness. Healthy subjects who are on a highfat, carbohydrate-free diet (Atkins diet, or the Inuits' traditional diet of seal and fish) also have no muscle weakness. So, the muscle weakness of the long-chain fat oxidation disorder patients cannot be explained by a decreased availability of acetyl-CoA fuel. Therefore, there must be some decrease in the tissues' ability to convert acetyl-CoA fuel to usable energy in patients with long-chain fat oxidation defects. Since there is no evidence of a defect in the respiratory chain of these patients, the defect lies probably in the processing of acetyl units by the CAC.

Proper operation of the CAC requires constant anaplerosis to compensate for physiological cataplerosis. When leakage of CAC intermediates is increased by an injury such as ischemia/reperfusion that damages cell membranes, the usual anaplerotic mechanisms can no longer compensate for the increased leakage. Anaplerosis from physiological concentrations of pyruvate $(0.05-0.1 \mathrm{mM}$ in plasma and even less within the cell) is a slow process because of the high $K_{\mathrm{m}}$ of pyruvate carboxylase for pyruvate $(0.4 \mathrm{mM})$. Substantial anaplerosis from pyruvate is observed at supraphysiological pyruvate concentrations (22-24). This explains why adding 1-3 mM pyruvate to the coronary blood improves the contractile ability of the heart (25-27) and decreases infarct size. This also explains why pyruvate improves the function of hearts perfused with AcAc (28). In long-chain fat oxidation disorders, rhabdomyolysis (CPK release in plasma) most likely results also in the leakage of catalytic intermediates of the CAC. When this occurs, it is likely that the rate of operation of the CAC is decreased in spite of ample supply of acetyl-CoA. Indeed, ongoing studies in catheterized pig hearts and in perfused rat hearts show rapid anaplerosis from low concentrations of $\left[\mathrm{U}_{-}{ }^{13} \mathrm{C}_{3}\right]$ propionate, $\left[5,6,7-{ }^{13} \mathrm{C}_{3}\right]$ heptanoate, and $\left[3,4,5-{ }^{13} \mathrm{C}_{3}\right] \mathrm{BKP}$, i.e., from precursors of $\left[\mathrm{U}^{-13} \mathrm{C}_{3}\right]$ propionyl-CoA (29). Anaplerosis from these substrates was demonstrated by the formation of M3 mass isotopomers of succinate. These animal data support our hypothesis that the beneficial effect of a dietary treatment of VLCAD deficiency with triheptanoin results from the anaplerotic character of this substrate.
In conclusion, the clinical and biological data of the present investigation, interpreted in the context of our animal studies, strongly support our hypothesis that the impairment in energy production in VLCAD deficiency results from an impairment in the oxidation of acetyl groups in the CAC. This occurs even in the presence of $\mathrm{C} 4$ ketone bodies derived from dietary even-chain MCT. The administration of heptanoate, a precursor of anaplerotic propionyl-CoA, results in a rapid and profound improvement in the cardiovascular and muscular status of the patient. Heptanoate and its derived C5 ketone bodies provide propionylCoA, which replenishes the pool of catalytic intermediates of the CAC. The resulting stimulations of acetyl-CoA oxidation, NADH production, and ATP regeneration improve muscle and heart contraction, restore membrane function, and decrease the loss of CAC intermediates.

The present report opens the way to the treatment with triheptanoin of each of the defects in long-chain fatty acid oxidation in children and adults. We are presently treating and evaluating 22 such patients. The clinical and biochemical data appear very promising in those with deficiencies of CPT I, translocase, CPT II, VLCAD, LCHAD, and trifunctional protein, as was suggested by the in vitro data with odd-carbon fatty acids in fibroblasts from patients with these disorders.

\section{Acknowledgments}

This work was supported by grants from Baylor University Medical Center's Research Foundation, the NIH (DK-35543), and the Cleveland Mt. Sinai Health Care Foundation. We thank SASOL GmbH and Mead Johnson Nutritionals for donating the triheptanoin and Portagen, respectively, used in this study. We also thank Christine Vianey-Saban, Robert A. Harris, Horst Schulz, Howard Sprecher, Patrick Finnegan, Jane Kao, and J.D. Cook, as well as C. Bazzarre, B. Garritson, J. Bennett, L. Alvarado, and S. Sharma, for fruitful scientific discussions and technical assistance with this investigation.

1. Dimauro, S., and Dimauro, P.M.M. 1973. Muscle carnitine palmitoyl transferase deficiency and myoglobinuria. Science. 182:929-931.

2. Engel, A.G., and Angelini, C. 1973. Carnitine deficiency of human skeletal muscle associated with lipid storage myopathy: a new syndrome. Science. 179:899-901.

3. Roe, C.R., and Ding, J.H. 2001. Mitochondrial fatty acid oxidation disorders. In The metabolic and molecular bases of inherited disease. 8th edition. C.R. Scriver, A.L. Beaudet, W.S. Sly, and D. Valle, editors. McGraw-Hill. New York, New York, USA. 2297-2326.

4. Sweetman, L. 2001. Newborn screening by tandem mass spectrometry: gaining experience. Clin. Chem. 47:1937-1938.

5. Saudubray, J.M., et al. 1999. Recognition and management of fatty acid oxidation defects: a series of 107 patients. J. Inherit. Metab. Dis. 22:488-502.

6. Bonnet, D., et al. 1999. Arrhythmias and conduction defects as presenting symptoms of fatty acid oxidation disorders in children. Circulation. 100:2248-2253

7. Leclerc, J., et al. 1995. Metabolism of R-beta-hydroxypentanoate and of beta-ketopentanoate in conscious dogs. Am. J. Physiol. 268:E446-E452.

8. Roe, C.R., Roe, D.S., Sharma, S., Zabot, M.T., and Vianey-Saban, C. 2001. Oxidation of unsaturated fatty acids by human fibroblasts with verylong-chain acyl-CoA dehydrogenase deficiency: aspects of substrate specificity and correlations with clinical phenotypes. Clin. Chim. Acta. 312:55-67. 
9. Roe, C.R., and Roe, D.S. 1999. Recent developments in the investigation of inherited metabolic disorders using cultured human cells. Mol. Genet. Metab. 68:243-247.

10. Gibson, K.M., et al. 2000. 2-Methylbutyryl-coenzyme A dehydrogenase deficiency: a new inborn error of L-isoleucine metabolism. Pediatr. Res. 47:1-4.

11. Powers, L., Osborn, M.K., Kien, C.L., Murray, R.D., and Brunengraber, H. 1995. Assay of the concentration and stable isotope enrichment of shortchain fatty acids by gas chromatography-mass spectrometry. J. Mass Spectrom. 30:747-754.

12. Souri, M., Aoyama, T., Hoganson, G., and Hashimoto, T. 1998. Verylong-chain acyl-CoA dehydrogenase subunit assembles to the dimer form on mitochondrial inner membrane. FEBS Lett. 426:187-190.

13. Vianey-Saban, C., et al. 1998. Mitochondrial very-long-chain acyl-coenzyme A dehydrogenase deficiency: clinical characteristics and diagnostic considerations in 30 patients. Clin. Chim. Acta. 269:43-62.

14. Roe, C.R., Wiltse, H.E., Sweetman, L., and Alvarado, L.L. 2000. Death caused by perioperative fasting and sedation in a child with unrecognized very long chain acyl-coenzyme A dehydrogenase deficiency. J. Pediatr. 136:397-399.

15. Sidbury, J.B., Chen, Y.T., and Roe, C.R. 1986. The role of raw starches in the therapy of type I glycogenosis. Arch. Intern. Med. 146:370-373.

16. Brown-Harrison, M.C., et al. 1996. Very long chain acyl-CoA dehydrogenase deficiency: successful treatment of acute cardiomyopathy. Biochem. Mol. Med. 58:59-65.

17. Cox, G.F., et al. 1998. Reversal of severe hypertrophic cardiomyopathy and excellent neuropsychologic outcome in very-long-chain acyl-coenzyme A dehydrogenase deficiency. J. Pediatr. 133:247-253.

18. Verhoeven, N.M., et al. 1998. Phytanic acid and pristanic acid are oxidized by sequential peroxisomal and mitochondrial reactions in human fibroblasts. J. Lipid Res. 39:66-74.
19. Bergmann, S.R., et al. 2001. Characterization of altered myocardial fatty acid metabolism in patients with inherited cardiomyopathy. J. Inherit. Metab. Dis. 24:657-674.

20. Bruce, M., et al. 2001. Glutamine supplementation promotes anaplerosis but not oxidative energy delivery in human skeletal muscle. Am. J. Physiol. Endocrinol. Metab. 280:E669-E675.

21. Rennie, M.J., Bowtell, J.L., Bruce, M., and Khogali, S.E. 2001. Interaction between glutamine availability and metabolism of glycogen, tricarboxylic acid cycle intermediates and glutathione. J. Nutr. 131(Suppl.):2488S-2490S.

22. Panchal, A.R., et al. 2000. Partitioning of pyruvate between oxidation and anaplerosis in swine hearts. Am. J. Physiol. Heart Circ. Physiol. 279:H2390-H2398

23. Comte, B., et al. 1997. A 13C mass isotopomer study of anaplerotic pyruvate carboxylation in perfused rat hearts. J. Biol. Chem. 272:26125-26131.

24. Comte, B., Vincent, G., Bouchard, B., and Des Rosiers, C. 1997. Probing the origin of acetyl-CoA and oxaloacetate entering the citric acid cycle from the $13 \mathrm{C}$ labeling of citrate released by perfused rat hearts. J. Biol. Chem. 272:26117-26124.

25. Mentzer, R.M., Jr., et al. 1989. Effect of pyruvate on regional ventricular function in normal and stunned myocardium. Ann. Surg. 209:629-633.

26. Lasley, R.D., Bunger, R., Zhou, Z., and Mentzer, R.M., Jr. 1994. Metabolically based treatment of stunned myocardium. J. Card. Surg. 9:469-473.

27. Bunger, R., et al. 1986. Pyruvate attenuation of hypoxia damage in isolated working guinea-pig heart. J. Mol. Cell. Cardiol. 18:423-438.

28. Russell, R.R., III, and Taegtmeyer, H. 1991. Changes in citric acid cycle flux and anaplerosis antedate the functional decline in isolated rat hearts utilizing acetoacetate. J. Clin. Invest. 87:384-390.

29. Kasumov, T., et al. 2002. Assay of the concentration and ${ }^{13} \mathrm{C}$-isotopic enrichment of propionyl-CoA, methylmalonyl-CoA, and succinyl-CoA by gas chromatography-mass spectrometry. Anal. Biochem. 305:90-96. 